\title{
The LHCb Upgrade Scintillating Fibre Tracker
}

\author{
Blake D. Leverington ${ }^{* \dagger}$ \\ Ruprecht-Karls-Universitaet Heidelberg, Germany \\ E-mail: b. leverington@cern.ch
}

\begin{abstract}
The Scintillating Fibre (SciFi) Tracker is designed to replace the current downstream tracking detectors in the LHCb Upgrade during 2018 (CERN/LHCC 2014-001; LHCb TDR 15). The operation and the results obtained from the data collected 2011 and 2012 demonstrate that the current detector is robust and functioning very well. However, the limit of $\mathscr{O}\left(1 \mathrm{fb}^{-1}\right)$ of data per year cannot be overcome without improving the detector. After 2018, it is planned to run with an increased luminosity of $2 \cdot 10^{33} \mathrm{~cm}^{-2} \mathrm{~s}^{-1}$ to collect up to $50 \mathrm{fb}^{-1}$ of data. This will be achieved using $25 \mathrm{~ns}$ bunch spacing with the average number of proton-proton interactions per bunch crossing $v=7.6$. Collecting data at this luminosity will only be possible if the detector is improved by increasing the readout of the front-end electronics to $40 \mathrm{MHz}$ and implementing a more flexible software-based triggering system that will increase the data rate as well as the efficiency. The increase in interactions per bunch crossing will result in an increased occupancy in the tracking detectors and will exceed the operational occupancy for the Outer Tracker. Here we present the SciFi Tracker as the replacement for the Outer and Inner Trackers.
\end{abstract}

The SciFi Tracker is based on $2.5 \mathrm{~m}$ long multi-layered ribbons from $\left(7 \cdot 10^{3} \mathrm{~km}\right)$ of $0.250 \mathrm{~mm}$ diameter scintillating fibre as the active medium and signal transport over 12 planes covering $375 \mathrm{~m}^{2}$. Cooled silicon photomultiplier (SiPM) arrays with 128 channels and $0.25 \mathrm{~mm}$ channel width are used as readout. The front-end electronics are designed to digitise the signals from the SiPMs with a custom ASIC chip, the PACIFIC, for the approximately 590k channels and reconstruct the track hit position within an on-board FPGA. Several challenges facing this detector will be presented regarding the precision construction of the large active detector components, the radiation hardness of the scintillating fibres and the SiPMs, the high density readout electronics, and the necessary cooling systems.

Technology and Instrumentation in Particle Physics 2014,

2-6 June, 2014

Amsterdam, the Netherlands

\footnotetext{
* Speaker.

†Presented on behalf of the LHCb SciFi Collaboration.
} 


\section{Introduction}

The LHCb detector [1] is built for indirect searches for New Physics (NP) through quantum loop induced processes, such as flavour-changing neutral currents, which are strongly suppressed in the SM, where precision is needed to observe these effects. These very rare decays and particle/anti-particle asymmetries are studied through the decay of known particles, particularly hadrons containing a $b$ or $c$ quark. These are compared with Standard Model (SM) predictions, looking for deviations resulting from additional NP particles or process. It is well known that these precision searches within $\mathrm{LHCb}$ also allow access to higher mass scales than the direct searches of ATLAS or CMS.

As of yet, in $\mathrm{LHCb}$, and at the $\mathrm{LHC}$ in general, no deviations from the SM have been observed. However, despite having many of the world's best measurements in some channels, LHCb is limited from improving on these measurements only by statistics and not systematic uncertainties. With this in mind an upgrade to the LHCb spectrometer has been proposed in order to collect 100 times more data than has been analysed so far in order to reduce the uncertainties of the measurements by a factor of ten such that they are equivalent to theoretical uncertainties [2, 3].

\section{The LHCb Detector, its limitations, and its upgrade}

The $\mathrm{LHCb}$ detector is a single-arm forward spectrometer covering the pseudo-rapidity range $2<\eta<5$, designed for studying particles containing $b$ or $c$ quarks, the production of which peaks in the forward region due to the boost provided by the asymmetric momentum of the colliding partons. A full description of the LHCb detector can be found in Ref. [1]

Interesting events are selected first by a hardware trigger stage (L0), based on transverse energy and momentum information from the calorimeter and muon stations. The L0 trigger reduces the primary visible collision rate $(\mu=0.7 \cdot v=1.8$ in 2012) down to a manageable $1 \mathrm{MHz}$ which is then sent to a second level software trigger stage (HLT) which applies a full event reconstruction where the events are written to storage. The LHCb luminosity is kept constant throughout the fill, but lower than the maximum possible, using a "luminosity levelling" technique, achieved through the partial separation of colliding beams at the interaction point of LHCb. This ensures a safe and efficient LHCb detector operation. In 2012 the L0 trigger rate has been brought to its maximum allowable value, such that a further increase in the rate of collected events is not possible. Data are currently recorded at an instantaneous luminosity of $4 \cdot 10^{32} \mathrm{~cm}^{-2} \mathrm{~s}^{-1}$, a factor of two above the design value. An increase in luminosity (and visible interactions per bunch crossing) would necessitate an increasing threshold cut on the energy and momentum in the L0 trigger to manage the $1 \mathrm{MHz}$ limitation. As a result, one does not see an increase in the yield of signal hadronic events that pass the trigger, though muon events will increase as they are read out at $40 \mathrm{MHz}$ into the trigger. To increase the data collection rate beyond the $1 \mathrm{MHz}$ limit requires a significant reworking of the trigger system, along with a replacement of all front-end electronics to a $40 \mathrm{MHz}$ readout.

For clarity, it should be stated that the LHCb luminosity upgrade is not tied to the LHC luminosity upgrade. 
In addition to the need for an improved trigger, another limitation of the current detector preventing it from running at higher luminosities is the Outer Tracker, the straw tube detector in the T-stations. It has been shown that the Outer Tracker can run at up to $25 \%$ occupancy $[2,3]$ before suffering a decrease in efficiency. However, in the LHCb Upgrade scenario, where the luminosity is five times higher, the occupancy will reach $40 \%$ and above. The parts of the detector affected require a replacement by one with a higher granularity such that the high density of tracks in the detector can be resolved.

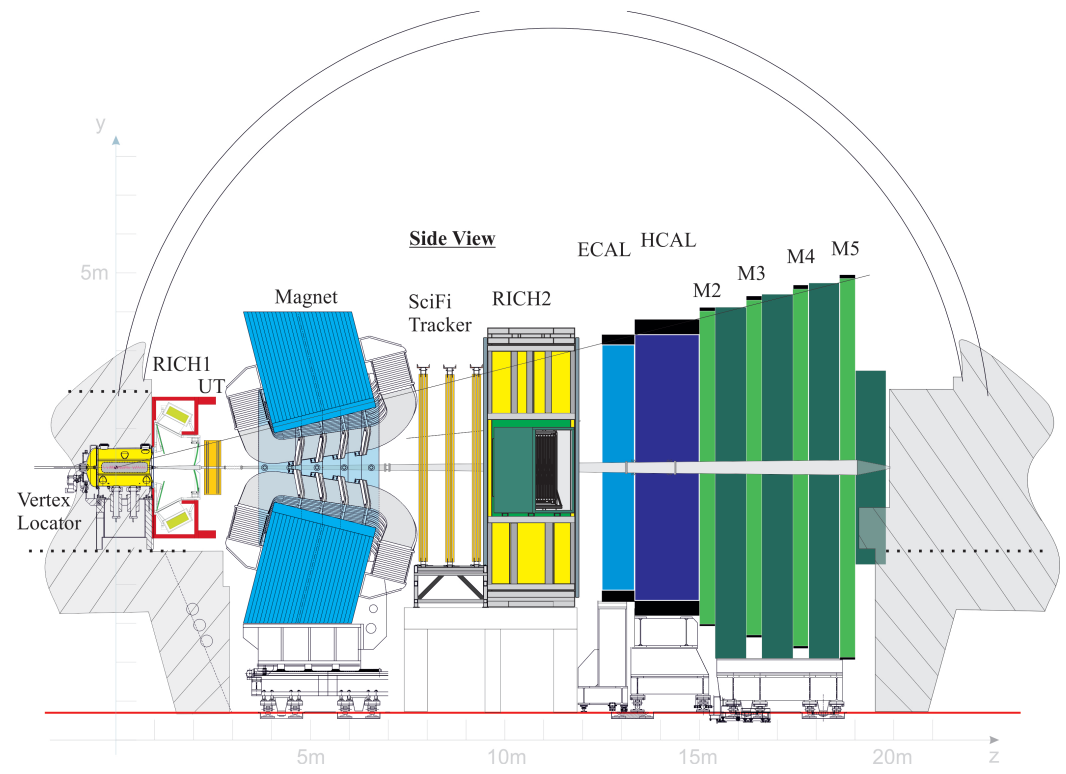

Figure 1: Schematic view of the LHCb upgrade detector. SciFi Tracker = Scintillating Fibre Tracker. [4]

Specific details regarding the upgrades to the other detectors can be found in the Letter of Intent [2], Framework TDR [3], and the specific detector Technical Design Reports available publicly. The most significant change to any of the detectors in the upgrade is replacing the current T-stations with a Scintillating Fibre ( $\mathrm{SciFi}$ ) based tracker, rather than modifying the existing detectors. This new tracker will be the focus of this Proceeding. A schematic of the upgraded LHCb detector is shown in Figure 1.

The upgraded detector will be able to collect at least $5 \mathrm{fb}^{-1}$ per year, with a highly flexible software trigger running at a readout rate of $40 \mathrm{MHz}$. This will increase the annual signal yields by a factor of about ten for muonic B decays and twenty or more for heavy-flavour decays to hadronic final states, as compared to those obtained by LHCb in 2011. A total integrated luminosity of $50 \mathrm{fb}^{-1}$ will be collected during the upgrade running.

\section{The Scintillating Fibre Tracker for the $\mathrm{LHCb}$}

The tracking detectors upstream and downstream of the LHCb dipole magnet provide a high precision estimate of the momentum of charged particles. This leads to a precise mass resolution for decayed particles. There are three downstream tracking (T-)stations: T1, T2, T3. They were 
designed to provide standalone pattern recognition with a high efficiency together with high resolution in the bending plane of the magnetic field. The tracking stations of the current detector consist of two separate sub-detectors: the Outer Tracker (OT) and the Inner Tracker (IT). The OT was constructed using 5-mm diameter straw detectors and covers around $99 \%$ of the $30 \mathrm{~m}^{2}$ detector surface. The IT is a silicon micro-strip detector covering an area of $0.35 \mathrm{~m}^{2}$ in the high track density region around the beam-pipe. Each station consists of four detection planes which provide measurements of the co-ordinates $\mathrm{X}, \mathrm{U}, \mathrm{V}$ and $\mathrm{X}$ with strips or straws orientated at $0+5,-5$ and 0 degrees, respectively, to the vertical axis.

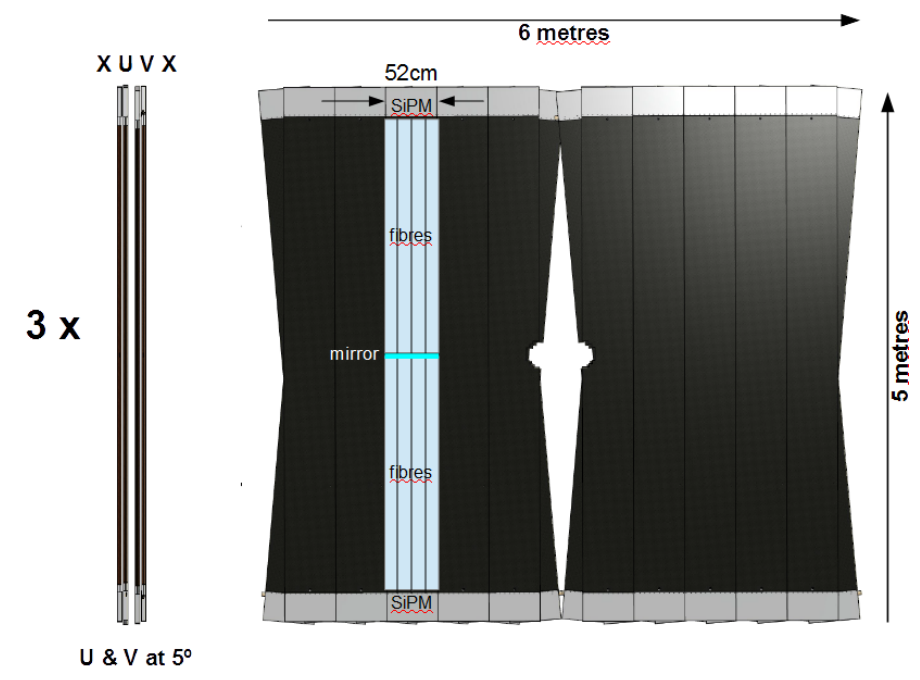

Figure 2: A sketch of 1 station of the SciFi tracker. Each station will have 4 layers $(X, U, V, X)$ oriented at $0,+5,-5$ and 0 degrees with respect to the vertical. Each layer will have 10-12 modules depending on the acceptance required and can open away from the beam-pipe at the centre. Each module will have $2.5 \mathrm{~m}$ long fibre mats, mirrored at the end nearest the centre, and read out with SiPMs at the far end outside of the acceptance.

The Scintillating Fibre ( $\mathrm{SciFi}$ ) Tracker replaces the OT and IT in the upgrade, with the same layer configuration and orientation as the OT, but with performance similar to the IT. The Technical Design Report (TDR) is now available publicly [4]. The SciFi Tracker has a channel width of $0.25 \mathrm{~mm}$ and a position resolution of approximately 60-70 micron in the bending plane of the magnet. It is constructed from $2.5 \mathrm{~m}$ long, $0.250 \mathrm{~mm}$ diameter, double clad scintillating fibres. The fibres consist of a polystyrene core, doped with two additional organic scintillators to improve the scintillation yield of the fibre, as well as shift the light to longer wavelengths improving the transmission of the light along the fibre. The baseline fibre for the experiment is the Kuraray SCSF-78MJ [5]. This fibre will typically produce a few thousand photons per $\mathrm{MeV}$ deposited, capture more than 5.35\% through total internal reflection at the cladding interfaces and has an average attenuation length greater than $3 \mathrm{~m}$ (the attenuation length is wavelength dependent). The wavelength spectra peaks at $435 \mathrm{~nm}$ near the source and extends into the green. This fibre type has a fast scintillation decay time of $2.8 \mathrm{~ns}$. Slower fibres would not work in this experiment as the bunch spacing in LHC will be $25 \mathrm{~ns}$, and the propagation time for a signal to travel $2.5 \mathrm{~m}$ is already approximately $15 \mathrm{~ns}$. 
Arrays of silicon photomultipliers (SiPMs; multi-pixel avalanche photodiodes operated in Geiger mode) are used as photodetectors, placed outside of the acceptance at one end of the scintillating fibres. The other end of the fibres will be mirrored to increase the light yield at the opposite end. Each SiPM array consists of 128 channels, $0.250 \mathrm{~mm}$ wide and approximately $1.3 \mathrm{~mm}$ tall with each channel consisting of 80-100 G-APD pixels. The small channel size, high photon detection efficiency $(\sim 40 \%)$, low voltage operation $(\sim 30-70 \mathrm{~V})$, relative ease of operation and immunity to magnetic fields makes this detector the only option for reading out a scintillating fibre detector of this size at the LHC. A sketch of the scintillation light produced in the fibres and detected in the SiPMs is shown in Figure 3. Typically, one detects 15-20 photoelectrons per particle track in the detectors before irradiation.
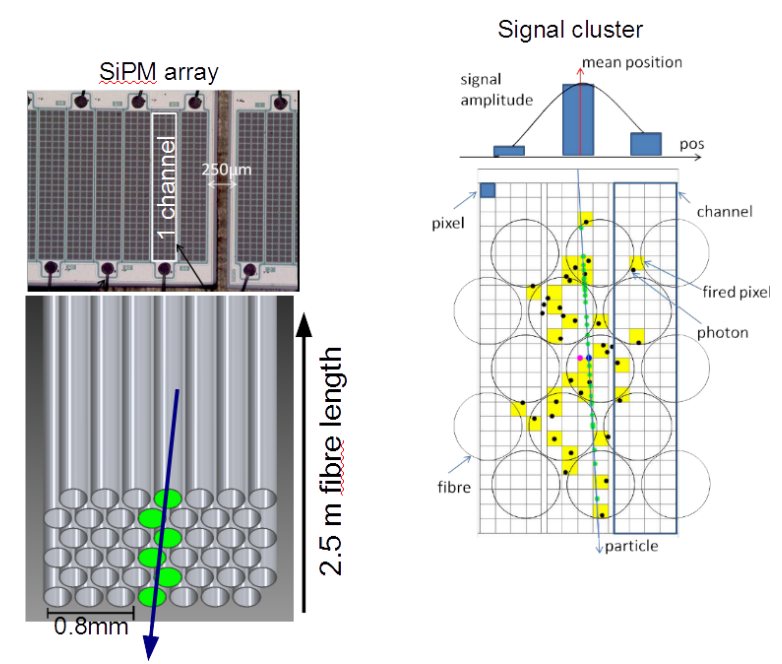

Figure 3: A sketch of the basic principle of the SciFi tracker. A charge particle passing through the fibre produces scintillation light (indicated in green). The light travels to the ends of the fibre where it is detected in the Silicon Photomultiplier arrays. The black dots indicate photons arriving at the detector. The yellow squares indicate the pixels that fire. The position of the track in the detector is determined by the (hit or charge) weighted mean of the channels where light is detected. [4]

\section{Detector Challenges}

Smaller detectors of this style have been constructed and operated before [6, 7], but never one of this size, covering approximately $375 \mathrm{~m}^{2}$, in the radiation environment of the the upgraded $\mathrm{LHCb}$ detector at LHC. The challenges faced constructing this detector and the impact of the radiation environment will be described below.

\subsection{Fibre mats}

As an individual scintillating fibre will only transmit on average a few photons, with Poissonlike fluctuations, the fibres must be packed into a dense matrix having 5-6 layers in order to produce a signal in the SiPM arrays at the ends of the fibre with a hit detection efficiency better than $98 \%$. Producing these fibre mats requires winding them on a threaded wheel approximately $0.8 \mathrm{~m}$ in 
diameter under light tension. Typically, $8 \mathrm{~km}$ of fibre is required to produce a fibre mat $2.5 \mathrm{~m}$ long, $13.5 \mathrm{~cm}$ wide and 6 layers thick. The position of the fibre is guided by a stepping motor and the thread on the wheel surface. Producing the mats is challenging as the fibre is quite delicate and the precision required is difficult relative to the size of the machines needed.

In addition, the fibre can come with defects from the manufacturer. Aside from ensuring consistent light yield and attenuation length between batches of scintillating fibre, the diameter of the fibre must be consistent. Surface defects of the fibre are typically in the form of "blobs", where the diameter of the fibre grows rapidly from the nominal $0.250 \mathrm{~mm}$ to diameters upwards of $0.40 \mathrm{~mm}$. This is normally a result of discontinuities in the extrusion process when producing the fibre.
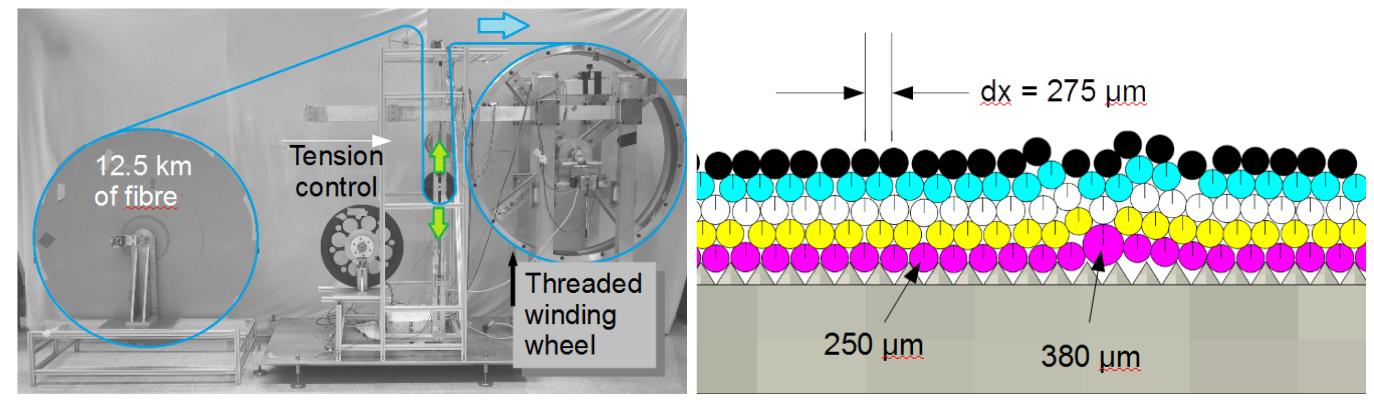

Figure 4: (left) A prototype winding machine. [4] (right) A sketch illustrating the problems caused by bumps on the fibre.

\subsection{Irradiation damage to fibres}

After the lifetime of the upgrade, it is expected that the scintillating fibres will receive up to $35 \mathrm{kGy}$ of ionizing radiation in the regions closest to the beam-pipe [8]. It is well known that plastic scintillating fibres will darken under irradiation, decreasing the transmission [9]. However, the literature describing this particular fibre is limited on the degree of damage in our radiation dose range. Three separate irradiation campaigns within the SciFi collaboration were undertaken to investigate the damage, irradiating the fibres with $24 \mathrm{GeV} / \mathrm{c}$ protons at the CERN-PS, $23 \mathrm{MeV}$ protons at the Karlsruhe Institute of Technology and in situ LHCb pit irradiations. The results are shown in Figure 5. The loss of transmission is rapid for low dose but the attenuation length flattens out at higher doses. Note the logarithmic horizontal axis. Given the highly localised region of high dose around the beam-pipe, it is expected that the fibre tracker will transmit enough of the scintillation light the the photodetectors to maintain a high hit efficiency, with a loss of only $40 \%$ of the signal in the worst region at the end of running. The scintillation production mechanism was not observed to be affected by irradiation.

\subsection{Irradiation damage to SiPMs}

Dark noise in the SiPMs is typically from thermal excitations of electrons into the conduction band of the silicon, causing the pixel to fire, similar to as if a single photon is absorbed. This happens typically at a rate of $100 \mathrm{kHz} / \mathrm{mm}^{2}$ for new generation SiPMs. However, this dark rate 

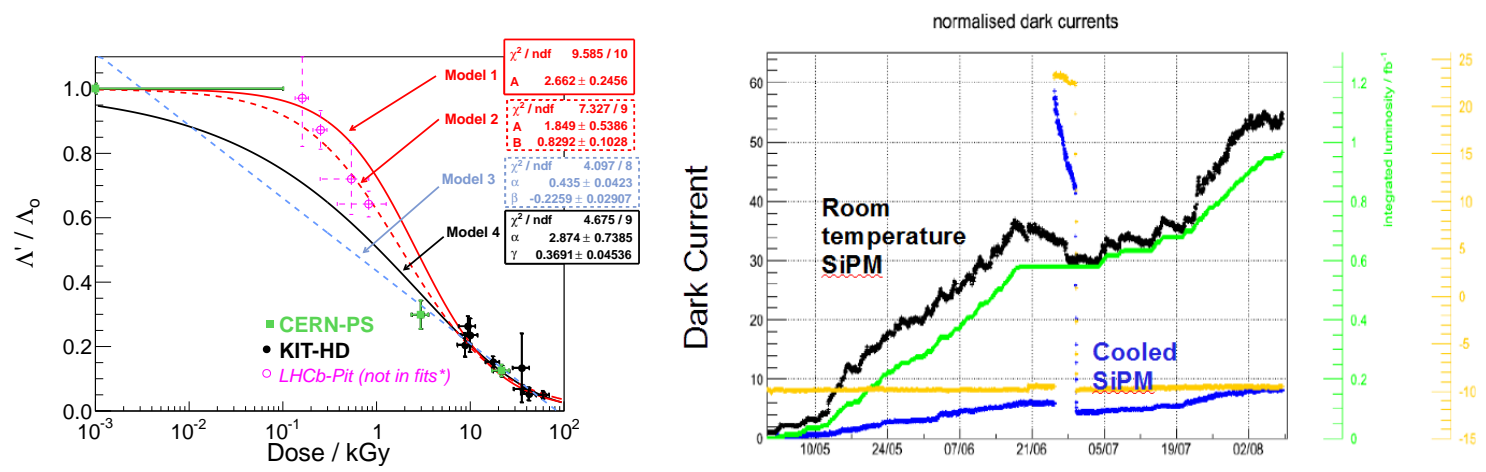

Figure 5: (left) The ratio of attenuation length after irradiation to before as a function of dose for scintillating fibres. The four curves fit to the data are an attempt to empirically model the damage as other studies have done [4]. (right) An example of increasing dark current of cooled (blue data) and non-cooled (blackdata) SiPMs shown with the increasing integrated luminosity (green data) in the LHCb pit. The yellow data are the temperature values. [10]

is strongly temperature dependent, decreasing by a factor of two for every 10 degrees Kelvin, approximately. Additionally, crosstalk between pixels cause adjacent neighbouring pixels to fire with a probability of a few percent. These multi-pixel dark noise signals look like low amplitude signals from tracks.

Compounding the problem is radiation damage to the silicon, increasing the single electron dark rate. The total integrated neutron flux expected in the upgrade at the location of the SiPMs is $\sim 1 \cdot 10^{12} \mathrm{~cm}^{-2} 1 \mathrm{MeV}$ equivalent neutrons in silicon. It is well known that the non-ionising energy losses (NIEL) in silicon photmultipliers results in damage to the lattice increasing the leakage current[11]. Neutron irradiation studies in the context of the LHCb are ongoing with new SiPM developments. Only 60-100 Gy of ionizing dose is expected. Given the large increase observed in dark count rate from the irradiation, the detectors will need to be cooled to -40 degrees Celsius during operation and regularly annealed at +40 degrees. This implies a great engineering challenge to prevent icing and condensation over $150 \mathrm{~m}$ of silicon detectors and their enclosures.

\subsection{Front-end electronics}

The SciFi Tracker will have over 590,000 SiPM channels that need to be digitised and integrated into the LHCb data system. A custom ASIC, the PACIFIC, is being developed to amplify and digitise the signals from the 128 channel SiPM arrays. It includes the analog signal processing and a 2 bit non-linear flash ADC for digitisation. The PACIFIC chip design incorporates very fast shaping (10ns) and the ability to cope with SiPMs of different gain and other varying signal properties. The power consumption is below $8 \mathrm{~mW}$ per channel. A set of thresholds must also be implemented within the ASIC such that low amplitude noise signals can be suppressed and the overall data rates can be managed. The digitised data is sent to an FPGA for hit reconstruction on-board the front-end card before being sent to the trigger and data farm.

\subsection{Detector construction}

The detector must be built with a very low material budget to minimize multiple scattering, 
which will degrade the low-momentum resolution. However, the detector must also be built strong such that it is stable in time and the position resolution does not degrade, especially for high momentum tracks. Constructing such a large detector, that also fulfils the roll of a much smaller silicon strip detector is very challenging. A rigid carbon-fibre/foam-core sandwich structure will be used (with the fibre mat in the middle of the sandwich) to stabilize the detector modules. This results in less than $1 \%$ of a radiation length (about $4 \mathrm{~mm}$ equivalent of plastic) per detector layer. Compared to the entire LHCb detector before the upgrade, the overall material budget has been reduced and a better momentum resolution is foreseen.

\section{Conclusion}

The SciFi Tracker is a challenging but exciting new detector for the LHCb Upgrade that is required to meet the scope of all the requirements for a fast and efficient spectrometer designed to discover new physics at the precision frontier. The challenges stated are not impossible to overcome, but require the participation of over 20 institutes from 10 countries. The resulting increase of a factor of 100 in data after 10 years will allow statistical uncertainties for several physics processes to be reduced to the theoretical uncertainties. Construction of this detector will begin in 2015 with installation set to begin in the middle of 2018 .

\section{References}

[1] LHCb collaboration, The LHCb Detector at the LHC, JINST 3 (2008) S08005.

[2] LHCb collaboration, Letter of Intent for the LHCb Upgrade, CERN-LHCC-2011-001, March 2011.

[3] LHCb collaboration, Framework TDR for the LHCb Upgrade, CERN-LHCC-2012-007, May 2012.

[4] LHCb collaboration, LHCb Scintillating Fibre Tracker Technical Design Report, CERN-LHCC-2014-001; LHCb TDR 15.

[5] Kuraray Plastic Scintillating Fibres, 2014, http://kuraraypsf.jp/psf/sf.html

[6] R. Greim. et al, A New Measurement of the Cosmic-Ray Flux Below 5GV Rigidity with the PERDaix Detector, Proceedings of the 20th ESA Symposium on European Rocket and Balloon Programmes and Related Research, Hyère, France, 2011

[7] P. von Doetinchem and others, PEBS - Positron Electron Balloon Spectrometer, Nucl. Instrum. Meth. A 581, 151-155, 2007

[8] N. Lopez March, M. Karacson, Radiation studies for the LHCb tracker upgrade, LHCb-PUB-2014-022, CERN-LHCb-PUB-2014-022, LHCb-INT-2013-003

[9] C. Zorn, A Pedestrians Guide To Radiation Damage In Plastic Scintillators, Nuclear Physics B (Proc. Suppl.) 32 (1993) 377-383.

[10] M. Deckenhoff, SiPM irradiation studies, SciFi meeting presentation, https://indico.cern.ch/event/174206/

[11] P. Bohn et al, Radiation damage studies of silicon photomultipliers, Nuclear Instruments and Methods in Physics Research Section A: Accelerators, Spectrometers, Detectors and Associated Equipment, 598, Issue 3, 2009, pp 722-736 